\title{
Chameleon-like elastomers with molecularly encoded strain-adaptive stiffening and coloration
}

\begin{abstract}
Mohammad Vatankhah-Varnosfaderani, ${ }^{1}$ Andrew N. Keith, ${ }^{1 *}$ Yidan Cong, ${ }^{1 *}$ Heyi Liang, ${ }^{2 *}$ Martin Rosenthal, ${ }^{3}$ Michael Sztucki, ${ }^{3}$ Charles Clair, ${ }^{4}$ Sergei Magonov, ${ }^{5}$ Dimitri A. Ivanov, ${ }^{6,7}$ Andrey V. Dobrynin, ${ }^{2}+$ Sergei S. Sheiko ${ }^{1} \dagger$
\end{abstract}

Active camouflage is widely recognized as a soft-tissue feature, and yet the ability to integrate adaptive coloration and tissuelike mechanical properties into synthetic materials remains elusive. We provide a solution to this problem by uniting these functions in moldable elastomers through the self-assembly of linear-bottlebrush-linear triblock copolymers. Microphase separation of the architecturally distinct blocks results in physically cross-linked networks that display vibrant color, extreme softness, and intense strain stiffening on par with that of skin tissue. Each of these functional properties is regulated by the structure of one macromolecule, without the need for chemical cross-linking or additives. These materials remain stable under conditions characteristic of internal bodily environments and under ambient conditions, neither swelling in bodily fluids nor drying when exposed to air.

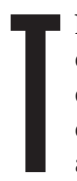

he mechanical and optical properties of biological tissues emerge from distinct physical origins but act in concert to serve living organisms such as chameleons, cephalopods, and amphibians (1-3). For example, initially soft and compliant tissues such as skin stiffen rapidly during deformation to prevent injury. Within narrow intervals of strain, their elastic moduli increase by several orders of magnitude at rates far beyond those observed for conventional elastomers, gels, and thermoplastics (Fig. 1, A to C). These tissues may also simultaneously convert white light into colorful patterns through constructive interference with light waves coherently scattered by periodic or quasi-periodic structures (Fig. 1D) (4). Collectively, these func- tions constitute defense and signaling mechanisms that have inspired the design of various biomimetic materials that either have tissuelike mechanics (5-11) or display structural colors (12-14). However, integrating both attributes into the molecular structure of a single material proves to be extremely challenging.

The mechanics of biological tissues arise from their composite nature, defined by the distinct mechanical response of two proteins, collagen and elastin (15). A scaffold of stiff collagen fibers resists deformation, and an interwoven elastin network ensures elastic recoil. This structural duet produces a characteristic, two-phase mechanical response (16), beginning with exponential stiffening and switching to a linear response ( $\sigma_{\text {true }} \sim \lambda$, where $\sigma_{\text {true }}$ is the true stress and $\lambda$ is the elongation ratio) halfway before the point of rupture, as exhibited by plotting differential modulus $\partial \sigma_{\text {true }} / \partial \lambda$ as a function of $\lambda$ (Fig. 1B). The

${ }^{1}$ Department of Chemistry, University of North Carolina at Chapel Hill, Chapel Hill, NC 27599, USA. ${ }^{2}$ Department of Polymer Science, University of Akron, Akron, $\mathrm{OH}$, 44325-3909, USA. ${ }^{3}$ European Synchrotron Radiation Facility, F-38043 Grenoble, France. ${ }^{4}$ Université de Haute Alsace, Laboratoire de Physique et Mécanique Textiles,

F-68093 Mulhouse Cedex, France. ${ }^{5}$ Scanning Probe Microscopy (SPM) Labs, Tempe, AZ 85283, USA. Institut de Sciences des Matériaux de Mulhouse-IS2M, CNRS UMR 7361, F-68057 Mulhouse, France. ${ }^{7}$ Lomonosov Moscow State University, Faculty of Fundamental Physical and Chemical Engineering, Leninskie Gory 1/51, 119991 Moscow, Russian Federation.

*These authors contributed equally to this work. †Corresponding author. Email: adobrynin@uakron.edu (A.V.D.); sergei@email.unc.edu (S.S.S.)
Fig. 1. Distinct mechanics and coloration of biological tissue. (A) A schematic of materials' true stress-elongation curves demonstrates the diversity of possible mechanical responses and the intense strain stiffening of initially soft tissue. Apparent Young's modulus $E_{0}$ is determined as the tangent of a stressstrain curve at $\lambda=1$. L, length of the sample at a given instant; $L_{0}$, initial length of the sample. (B and $\mathbf{C}$ ) Differential modulus of biological tissues and synthetic materials (tables S6 and S7). bb, bottlebrush; Synt., synthetic; PU/AM, polyurethane/acrylamide hydrogel. (D) Color variation in the skin of a male panther chameleon changing from a relaxed to an excited state. [Adapted from (1)] (Insets) Transmission electron microscopy images of the guanine nanocrystal lattice from the same individual in the corresponding states.
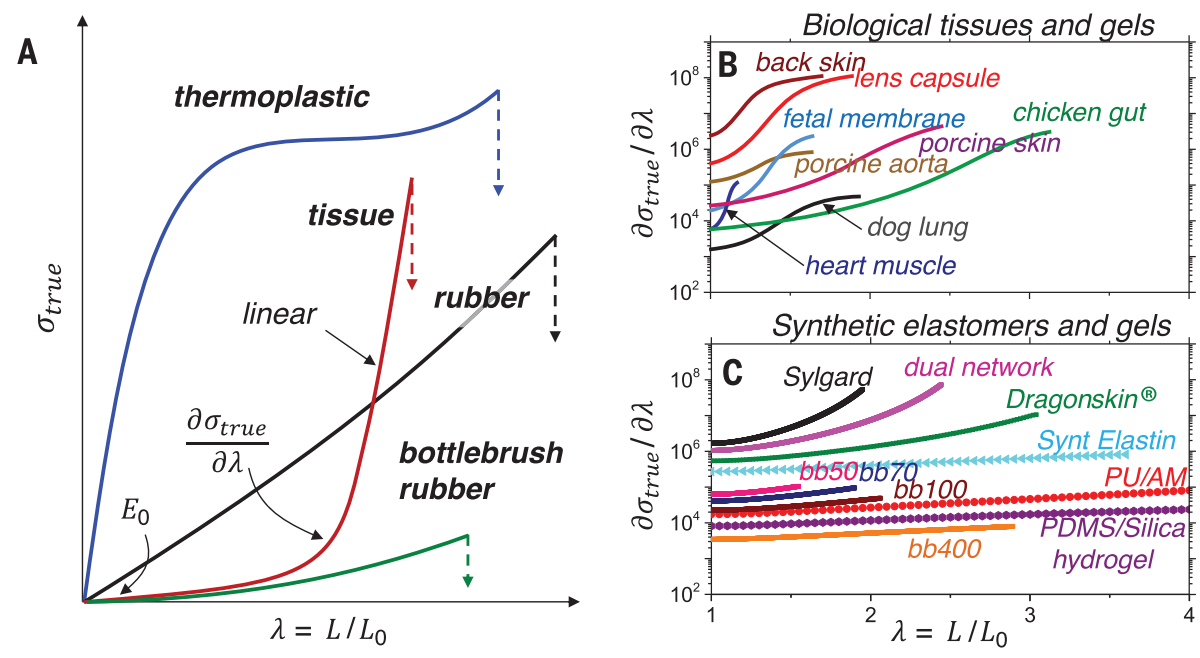

D
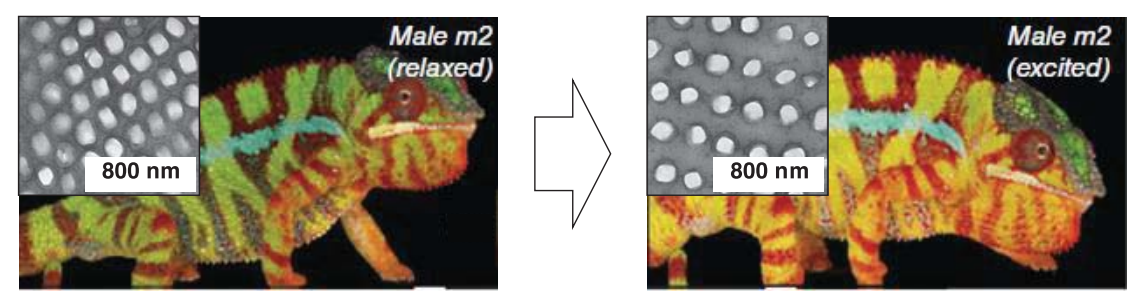
resulting sigmoid shape of the $\partial \sigma_{\text {true }} / \partial \lambda$ curves contrasts with the steady increase in stiffness displayed by synthetic elastomers and gels (Fig. 1C). Although various molecular and macroscopic constructs implement the basic principles of strain stiffening (6-11), none replicate tissue's deformation response completely and precisely. For example, various silicone rubbers, such as Ecoflex and Dragon Skin, which are widely used in orthotics and cinematography (17), have skinlike softness but lack the strain-stiffening characteristics of skin (Fig. 1C). Polymeric gels are similarly incapable of replicating tissue mechanics (Fig. 1C) and further suffer from solvent leakage upon deformation (18).

We simultaneously enhanced softness and strain stiffening in recent work by employing brushlike architecture in solvent-free elastomers (Fig. 1C) (19). The attachment of side chains to network strands yielded a dual mechanical effect: Moduli were reduced to $100 \mathrm{~Pa}$ via chain disentanglement, and strain stiffening increased by an order of magnitude via strand extension due to side-chain steric repulsion (20). Although this effect enables mechanical replicas of gel-like tissues such as lung and jellyfish (5), the strain stiffening of these systems pales when compared with that of soft connective tissues like skin. In parallel, various chromogenic polymers have been created (21-23), but they fail to incorporate tissuelike mechanical properties. This conventional divide presents a material design challenge aimed at mimicking skin tissue-soft on touch, stiff upon deformation, and colored for appeal or camouflage.

We report the design of chromogenic elastomers, formed by microphase separation of linearbottlebrush-linear ABA triblock copolymers (Fig. 2A), that are solvent free, supersoft (apparent Young's modulus $E_{0} \sim 10^{3}$ to $10^{5} \mathrm{~Pa}$ ), and strongly strain stiffening $\left(E_{0}^{-1} \partial \sigma_{\text {true }} / \partial \lambda \sim 1\right.$ to $\left.10^{2}\right)$. We explored the symbiosis of two blocks that are both chemically and physically distinct: (i) flexible linear chains that aggregate into rigid domains and (ii) stiff bottlebrush strands that form a supersoft matrix. This interplay of the attributes of rigid-while-flexible and soft-while-stiff, which are oxymoronic upon first glance, is actually representative of the distinctive potential of architectural control over materials' mechanical and optical properties. Elucidating how both building blocks not only play individual roles in this control but also synergize with each other to enhance biomimetic performance is a central feature of this study. Specifically, we show that aggregation of linear blocks yields physical networks, and bottlebrush strands provide these networks with a low modulus. Further, the strong segregation of the chemically dissimilar blocks (24) augments the architectural prestrain of the bottlebrush blocks
(20) and thereby markedly improves the networks' strain-stiffening characteristics (fig. S15). These effects enable replication of the mechanical response of porcine skin and achieve substantial progress toward replicating that of human skin, as discussed below.

To validate this concept, we have synthesized several series of linear-bottlebrush-linear ABA triblock copolymers with different degrees of polymerization (DPs; represented as $n$ ) of the bottlebrush backbone ( $n_{\mathrm{bb}} \approx 300$ to 1800) and identical DPs of the polydimethylsiloxane (PDMS) side chains $\left(n_{\mathrm{sc}}=14\right)$ (Fig. 2A, fig. S1 to S3, and table S1). Each series contains molecules with different DPs of the linear A block ( $n_{\mathrm{A}} \approx 80$ to 1200 ) corresponding to A-block volume fractions $\left(\phi_{A}\right)$ of 0.03 to 0.3 , where the A block may be poly(methyl methacrylate) (PMMA), poly(benzyl methacrylate) (PBzMA), or poly(oligo(ethylene glycol) monomethyl ether methacrylate) [P(OEOMA)]. For physical tests, thin films were prepared by solution casting. During solvent evaporation, microphase separation results in thermoplastic elastomers (plastomers) with characteristic coloration produced by constructive interference with light waves reflected by domain interfaces (Fig. 2B and movie S1). Both color and mechanical properties depend on plastomer morphology, which was characterized by differential scanning calorimetry (DSC) and atomic force microscopy (AFM)
Fig. 2. Structural coloration of plastomers.

(A) Self-assembly of linear-bottlebrush-linear ABA triblock copolymers yields physical networks-A domains of linear blocks embedded in a B matrix of bottlebrush strands, where $n_{\mathrm{A}}, n_{\mathrm{bb}}$, and $n_{\mathrm{sc}}$ are degrees of polymerization (DPs) of the linear block, the bottlebrush backbone, and the bottlebrush side chains, respectively. The microphase-separated structure is described in terms of the interbrush distance $\left(d_{1}\right)$, the diameter of the spherically shaped PMMA domains $\left(d_{2}\right)$, and the interdomain distance $\left(d_{3}\right)$. $Q$, aggregation number. (B) Structural coloration in a broad spectral range is typically observed during solvent evaporation and is indicative of decreasing distance between A domains (movie S1). (C) AFM height micrographs corroborate microphase separation of PMMA-bottlebrush backbone PDMS (bbPDMS)-PMMA plastomers with identical $n_{\mathrm{bb}}$ values of 938 , varied linear PMMA DPs, and a designated PMMA volume fraction $\left(\phi_{A}\right)$ (series M900- $x$, where $x$ is any number, in table S1). (D) USAXS patterns of the plastomers represented above display characteristic length scales as depicted in (A) and summarized in table S4. a.u., arbitrary units; q, magnitude of the scattering wave vector. (E) Concentrated solutions (25 weight \%) of the corresponding plastomers. Evaporation of turquoise solution 4 (M900-4) yields a blue film. v\%, volume \%; NA, not applicable.

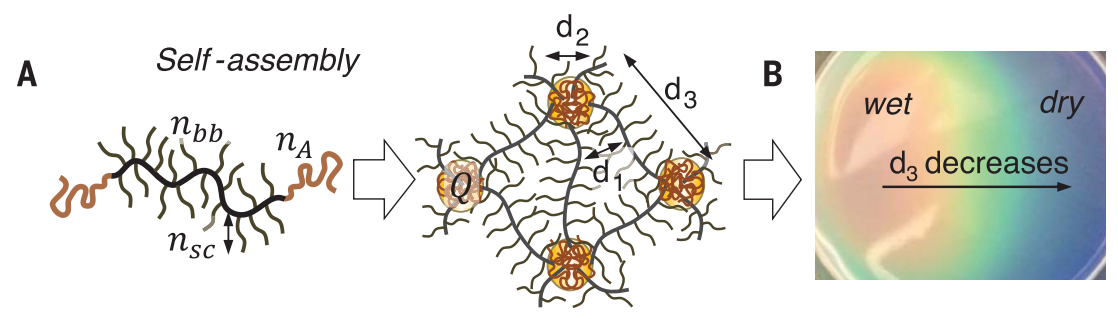

C
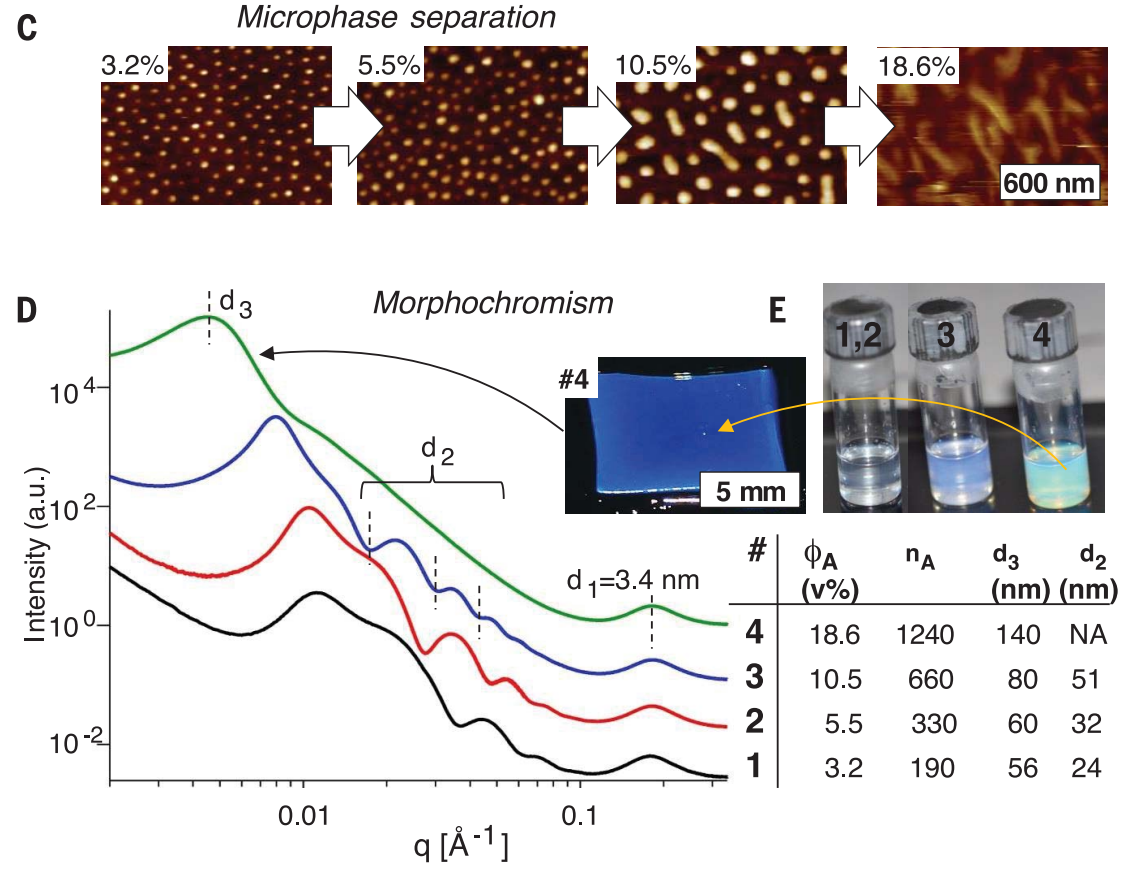
(Fig. 2C, figs. S5 to S7, and table S2) and ultrasmall-angle x-ray scattering (USAXS) (Fig. 2D and fig. S17). This combination of techniques provided the interbrush distance $\left(d_{1}=3.4 \mathrm{~nm}\right)$, the domain diameter $\left(d_{2}=20\right.$ to $\left.40 \mathrm{~nm}\right)$, the aggregation number $(Q \cong 300$ to 1000$)$, and the interdomain distance $\left(d_{3}=40\right.$ to $\left.150 \mathrm{~nm}\right)$ as summarized in table S4 for different copolymer compositions.

Corresponding analysis revealed that plastomers' block dimensions $\left(n_{\mathrm{bb}}, n_{\mathrm{A}}\right.$, and $\left.\phi_{\mathrm{A}}\right)$ strongly affect their mechanical properties (Fig. 3, A and $\mathrm{B}$, and figs. S8 to S11), which include a low initial modulus $\left(E_{0}=4\right.$ to $\left.50 \mathrm{kPa}\right)$ (table $\mathrm{S} 1$ ); intense strain stiffening, depicted as a 10- to 100-fold increase of the differential modulus within a short strain interval (Fig. 3, C and D); and the characteristic sigmoid shape of the $\partial \sigma / \partial \lambda$ curves, which is markedly similar to that for biological tissues (Fig. 1B). All of these features are a manifestation of a two-phase deformation process, which starts with the extension of architecturally prestrained bottlebrush network strands (the elastic regime) and proceeds with the uncoiling of linear chains in A domains (the yielding regime) (figs. S20 and S23). Unlike that in the elastic phase of deformation, yielding-phase stress depends on strain rate (fig. S13) and develops small (<10\%) hysteresis in loading-unloading cycles (fig. S14). All three features (reversibility, strain-rate dependence, and hysteresis) are also observed via yielding of collagen assemblies in biological tissues, which provides energy dissipation and additional extensibility $(9,25)$. Even though plastomers have a completely different structure and distinct deformation mechanisms from biological tissues, they display a tissuelike mechanical response. Although one may intuitively presume that the prestrain of the B blocks would impair network extensibility, uncoiling of the flexible A blocks compensates for this inherent characteristic. In other words, the A domains serve as reservoirs of untapped network extension and yield synergy that is muted in all-linear ABAs, wherein the absence of prestrain diminishes the phaseseparation enhancement of strain stiffening (fig.
A

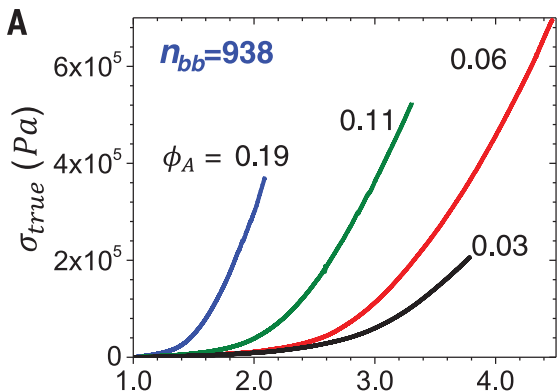

C

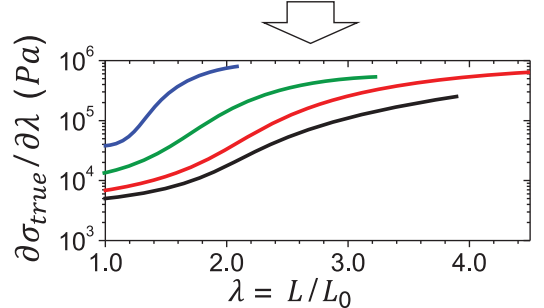

$\mathbf{E}$

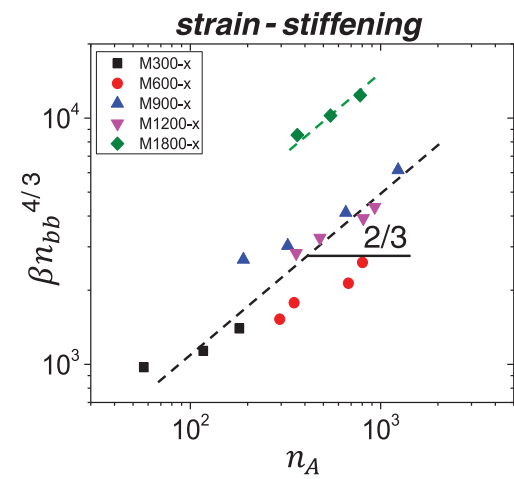

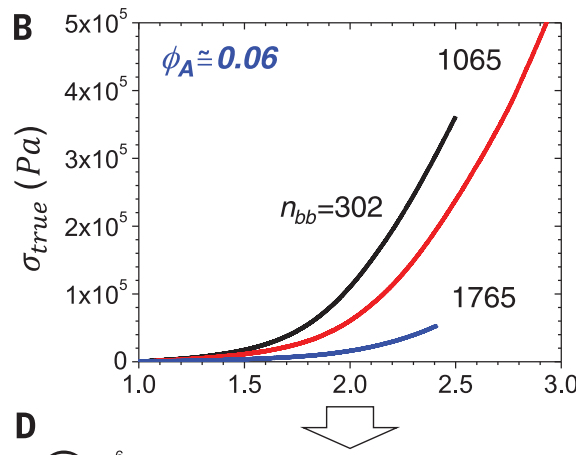

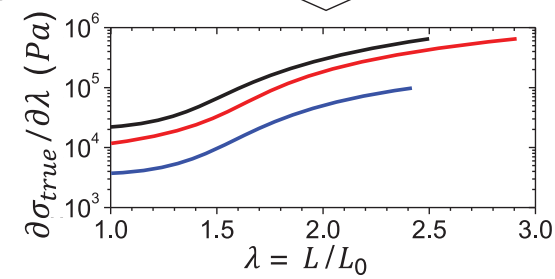

$\mathbf{F}$

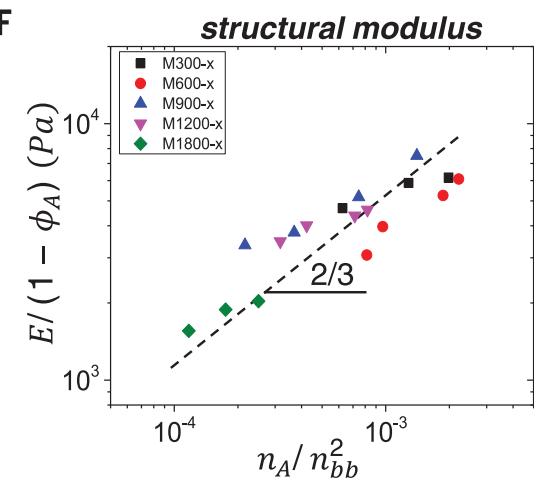

Fig. 3. Mechanical properties of plastomers as a function of block copolymer composition. (A) Stress-strain curves of PMMA-bbPDMS-PMMA plastomers with identical $n_{\mathrm{bb}}$ values of 938 and varied A-block volume fractions $\left(\phi_{A}\right)$ (series M900-x in table S1) measured upon uniaxial extension in the elastic regime (constant strain rate, $0.005 \mathrm{~s}^{-1}$; temperature, $25^{\circ} \mathrm{C}$ ) (fig. S4).

(B) Stress-strain curves of PMMA-bbPDMSPMMA plastomers with different $n_{\mathrm{bb}}$ values but similar $\phi_{A}$ values of $\sim 0.06$. (C and $\mathbf{D}$ ) Differential moduli for plots in (A) and (B), respectively. (E) Normalized strain-stiffening parameter $\beta$ increases with the DP of linear PMMA blocks as $\beta n_{\mathrm{bb}}^{4 / 3} \sim n_{\mathrm{A}}^{2 / 3}$. E and $\beta$ values are obtained by fitting stress-strain curves using eq. S6.18 in the elastic phase of network deformation (fig. S12). (F) Structural Young's modulus decreases with $n_{\mathrm{bb}}$ and increases with $n_{\mathrm{A}}$ as $E \sim n_{A}^{2 / 3} n_{b b}^{-4 / 3}$. The data in (E) and (F) are means with SDs of 5 to $15 \%$ (table S1). (G) Universal plot of normalized differential modulus $E_{0}^{-1} \partial \sigma_{\text {true }} / \partial \lambda$ versus $\sigma_{\text {true }} / \sigma^{*}$, where $\sigma^{*}=E^{1 / 3} E_{0}^{2 / 3} / 3 \beta^{2 / 3}$ is the crossover stress between the linear and strain-stiffening regimes (eq. S6.27 and table S3).
G

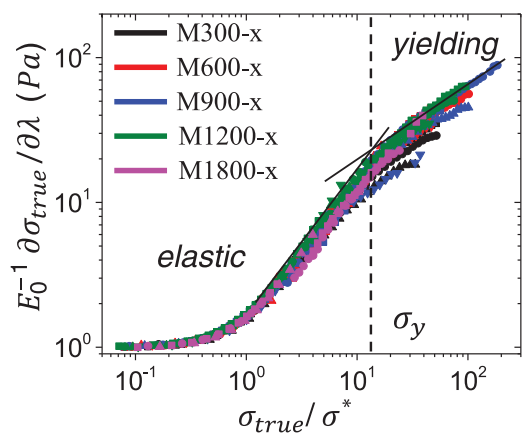




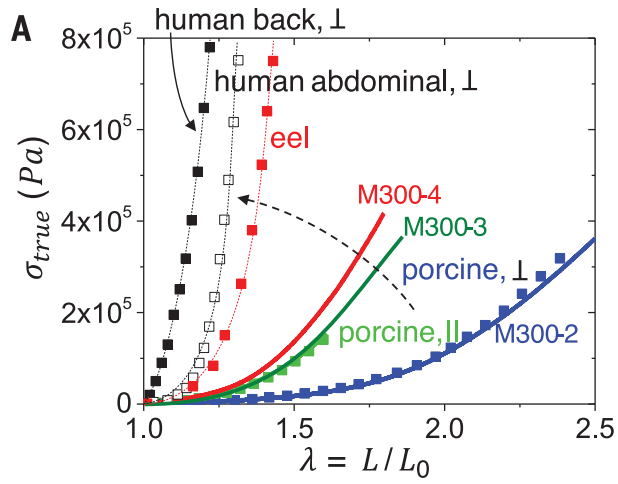

B

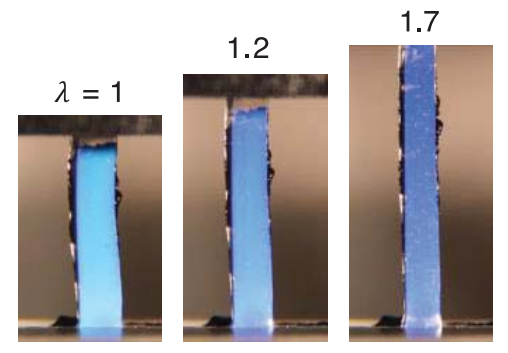

C
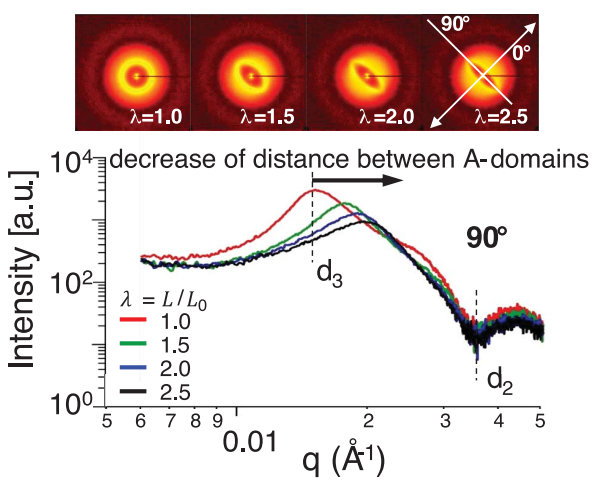

D
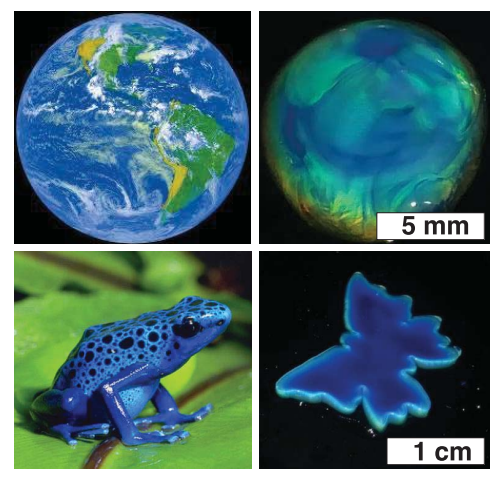

Fig. 4. Mimicking skin tissue. (A) True stress-elongation curves for assorted skin tissues (human back, human abdominal, eel, and porcine in table S7 and fig. S24) (squares) and M300-x plastomers cast from tetrahydrofuran with identical $n_{\mathrm{bb}}$ values of 302 and varied DPs of the linear PMMA block (lines). Current progress toward mimicking human skin is highlighted by the dashed arrow. $\perp$, tissue was measured perpendicularly to the spine; $\|$, tissue was measured parallel to the spine. (B) An observed color alteration from turquoise to dark blue during uniaxial stretching of a 2-mm-thick (G900-1) P(OEOMA)-bbPDMS-P(OEOMA) film (movie S2).

(C) Two-dimensional USAXS patterns corresponding to different extension ratios exemplified for M300-2. Sections of the USAXS patterns are measured perpendicularly (at a $90^{\circ}$ angle) to the stretching direction at different elongation ratios. Elongation shifts the main interference maximum, which suggests a shortening between a PMMA domain's nearest neighbors $\left(d_{3}\right.$ in Fig. 2A). Deformation has no effect on the A-domain form factor $\left(d_{2}\right)$. (D) (Top) Satellite image of Earth (left) and a drop of P(OEOMA)-bbPDMS-P(OEOMA) solution in toluene during drying (right). (Bottom) Blue poison dart frog (left) and a thin butterfly cutout of PBzMA-bbPDMS-PBzMA plastomer B1000-2 (table S1) with edges swollen with linear PDMS for contrast on a glass substrate (right). The back side of the glass substrate was painted black to enhance reflectance.

S16), and all-brush ABAs, wherein added architectural prestrain impedes the overall extensibility of the material (26-29).

For the elastic phase of deformation, we analyzed the stress-strain curves using a constitutive network deformation model (eq. S6.18), which has been validated for various polymer networks, including biological gels $(5,19,30)$. This model is described by two parameters: (i) structural Young's modulus, a measure of cross-link density (eq. S6.22), and (ii) the strain-stiffening parameter $(\beta)$, a measure of strand extension defined as $\beta \equiv\left\langle R_{\text {in }}^{2}\right\rangle / R_{\max }^{2}$, where $R_{\max }$ is the contour length of a fully extended strand and $\left\langle R_{\text {in }}^{2}\right\rangle$ is the mean square end-to-end distance of strands in asprepared elastomers (eq. S6.19). Parameter $\beta$ is controlled by finite extensibility of network strands, with the lower and upper bounds cor- responding to networks with coiled $\left(R_{\mathrm{in}} \ll R_{\max }\right.$, $\beta \rightarrow 0)$ and extended $\left(R_{\text {in }} \cong R_{\max }, \beta \rightarrow 1\right)$ strands, respectively. For comparison, typical linear-chain elastomers, including linear ABAs (26), are characterized by $\beta \cong 0.01$ (fig. S16). Although bottlebrush covalent networks allow for a substantial increase, up to $\beta \cong 0.3$ (table S6) (19), this value is nonetheless notably lower than those in the range for tissue, where $\beta \cong 0.5$ to 0.9 (table S7). Microphase separation in our plastomers results in additional strand extension, with $\beta \cong 0.3$ to 0.8 (table $\mathrm{S} 1$ ), a range that favorably overlaps with values for tissues, such as $\beta=0.69$ (lung), $\beta=0.75$ (brain), $\beta=0.78$ (skin), and $\beta=0.75$ (blood vessel) (table S7).

To highlight the corresponding capacity for predictably controlling strain stiffening, table $\mathrm{S} 1$ summarizes the molecular and mechanical pa- rameters of the studied plastomers. The strainstiffening parameter $(\beta)$ follows the theoretically predicted scaling relation $\beta \sim n_{\mathrm{A}}^{2 / 3} n_{\mathrm{bb}}^{-4 / 3}$ (Fig. 3E), which originates from chain extension $\left\langle R_{\text {in }}^{2}\right\rangle \sim$ $n_{\mathrm{A}}^{2 / 3} n_{\mathrm{bb}}^{2 / 3}$ (eqs. S6.12 and S6.17), as is well documented for block copolymer systems in the strong segregation limit (24). Similarly, the structural modulus follows $E \sim\left(1-\phi_{\mathrm{A}}\right) n_{\mathrm{A}}^{2 / 3} n_{\mathrm{bb}}^{-4 / 3} \sim\left(1-\phi_{\mathrm{A}}\right) \beta$ (Fig. 3F and fig. S21), which originates from prestretching of the bottlebrush strands upon microphase separation (eq. S6.22). The established structure-property correlations therefore allow for universal presentation of the differential modulus as a function of stress (Fig. 3G). The observed deviation of $\partial \sigma_{\text {true }} / \partial \lambda$ curves in the yielding regime occurs at different elongations because of the difference in finite extensibility of the various ABA network strand lengths (Fig. 3, C and D). Because chain withdrawal proceeds at constant force $f\left(f \sim \sigma_{\text {eng }}\right.$, where $\sigma_{\text {eng }}$ is engineering stress), the true stress in the yielding regime scales linearly with $\lambda$ as $\sigma_{\text {true }} \sim \lambda$ (fig. S23). In other words, the stress-strain behavior of different plastomers follows a universal trend that is architecturally controlled.

Tensile stress-strain curves for assorted skin tissues, like those for plastomers, exhibit broad variation of mechanical properties defined by low modulus $E$ ( 0.4 to $12 \mathrm{kPa}$ ) and substantial strain stiffening, with $\beta \cong 0.5$ to 0.9 (Fig. $4 \mathrm{~A}$ and table S7). This parallel is exemplified by comparing the stress-strain curves of samples M300-2 and M300-3 with those of porcine skin measured perpendicularly and parallel to the spine, respectively. The precise overlap thereof demonstrates that plastomers can replicate the deformation response of certain strain-stiffening tissues completely and precisely. Furthermore, elongation results in a blue shift of sample color (Fig. 4B and movie S2) because of the corresponding decrease in interdomain distance $\left(d_{3}\right)$ (Fig. $\left.4 \mathrm{C}\right)$. This deformation does not affect domain size $\left(d_{2}\right)$ and underlines the robust nature of the physical network. Similarly, solvent swelling results in omnidirectional expansion between domains, with an observed shift in color (Fig. 2B and movie S1), which is consistent with USAXS (fig. S18A) and reflectance (fig. S18B) measurements of plastomers in selective solvents. Inhomogeneous swelling (or drying) thus demonstrates spectacular visuals that resemble the optical complexity of natural systems such as Earth and blue poison dart frogs (Fig. 4D). This color shift also highlights how the same network structure that lends plastomers their mechanical characteristics also imbues them with adaptive structural coloration.

In conclusion, we have established that the selfassembly of linear-bottlebrush-linear triblock copolymers (plastomers) empowers the integration of strain-adaptive stiffening and strain-induced coloration. We have further demonstrated that this class of materials enables replication of the mechanical response of strongly strain-stiffening tissues, with porcine skin as an example, and we have shown that replication of the response of human skin is within reach. We hope to expand 
this materials design platform by achieving independent control over mechanical and coloration responses.

\section{REFERENCES AND NOTES}

1. J. Teyssier, S. V. Saenko, D. van der Marel, M. C. Milinkovitch, Nat. Commun. 6, 6368 (2015)

2. L. M. Mäthger, E. J. Denton, N. J. Marshall, R. T. Hanlon, J. R. Soc. Interface 6 (suppl. 2), S149-S163 (2009).

3. I. C. Cuthill et al., Science 357, eaan0221 (2017).

4. J. P. Vigneron, P. Simonis, Physica B 407, 4032-4036 (2012).

5. M. Vatankhah-Varnosfaderani et al., Nature 549, 497-501 (2017)

6. S. C. Grindy et al., Nat. Mater. 14, 1210-1216 (2015).

7. B. Yu et al., Nat. Mater. 15, 911-918 (2016).

8. A. S. So, Tayi, F. Güder, G. M. Whitesides, Adv. Funct. Mater. 24, 7197-7204 (2014).

9. W. Yang et al., Nat. Commun. 6, 6649 (2015).

10. M. Jaspers et al., Nat. Commun. 8, 15478 (2017)

11. E. Ducrot, Y. Chen, M. Bulters, R. P. Sijbesma, C. Creton, Science 344, 186-189 (2014)

12. K. Yu, T. Fan, S. Lou, D. Zhang, Prog. Mater. Sci. 58, 825-873 (2013)

13. S. A. Morin et al., Science 337, 828-832 (2012).

14. Y. Zhao, Z. Xie, H. Gu, C. Zhu, Z. Gu, Chem. Soc. Rev. 41 , 3297-3317 (2012)

15. G. A. Holzapfel, in Handbook of Material Behavior Models, J. Lemaitre, Ed. (Academic Press, 2001), pp. 1057-1071.

16. C. Storm, J. J. Pastore, F. C. MacKintosh, T. C. Lubensky, P. A. Janmey, Nature 435, 191-194 (2005).

17. T. Ranzani, G. Gerboni, M. Cianchetti, A. Menciassi, Bioinspir. Biomim. 10, 035008 (2015).

18. Y. Li et al., Sci. Rep. 5, 17017 (2015).

19. M. Vatankhah-Varnoosfaderani et al., Adv. Mater. 29, 1604209 (2017).
20. S. S. Sheiko et al., Nature 440, 191-194 (2006).

21. J. H. Lee et al., Adv. Mater. 26, 532-569 (2014).

22. A. L. Liberman-Martin, C. K. Chu, R. H. Grubbs, Macromol. Rapid Commun. 38, 1700058 (2017)

23. Y. Fu, C. A. Tippets, E. U. Donev, R. Lopez, Wiley Interdiscip. Rev. Nanomed. Nanobiotechnol. 8, 758-775 (2016).

24. F. S. Bates, G. H. Fredrickson, Annu. Rev. Phys. Chem. 41, 525-557 (1990)

25. B. Depalle, Z. Qin, S. J. Shefelbine, M. J. Buehler, J. Mech. Behav. Biomed. Mater. 52, 1-13 (2015).

26. J. M. Yu, Ph. Dubois, Ph. Teyssie, R. Jerome, Macromolecules 29, 6090-6099 (1996).

27. K. A. Erk, K. J. Henderson, K. R. Shull, Biomacromolecules 11 1358-1363 (2010)

28. R. Verduzco, X. Li, S. L. Pesek, G. E. Stein, Chem. Soc. Rev. 44, 2405-2420 (2015).

29. C. M. Bates, A. B. Chang, N. Momčilović, S. C. Jones, R. H. Grubbs, Macromolecules 48, 4967-4973 (2015).

30. J.-M. Y. Carrillo, F. C. MacKintosh, A. V. Dobrynin, Macromolecules 46, 3679-3692 (2013)

\section{ACKNOWLEDGMENTS}

We thank K. Matyjaszewski for illuminating discussions; Q. Li, W. Daniel, and B. Morgan for their assistance in mechanical tests; and M. Everhart for reviewing the manuscript. Funding: This work made use of the Cary 5000 ultraviolet-visible-nearinfrared (UV-Vis-NIR) with Agilent diffuse reflectance accessory instrument in the University of North Carolina (UNC) Energy Frontier Research Center (EFRC) instrumentation facility established by the UNC EFRC Center for Solar Fuels, an EFRC funded by the U.S. Department of Energy, Office of Science, Office of Basic Energy Sciences, under award number DE-SC0001011. USAXS measurements were performed at the ID02 beamline, European
Synchrotron Radiation Facility. We gratefully acknowledge funding from the NSF (DMR 1407645, DMR 1436201, and DMR 1624569) and from the Russian Science Foundation (project no. 16-13-10369), Author contributions: M.V.-V. designed, synthesized, and characterized the monomers, block copolymers, and polymer networks; A.N.K. performed synthesis of block copolymers, mechanical tests, and AFM experiments; Y.C. synthesized PBzMA-bottlebrush backbone PDMS-PBzMA plastomers and studied structural coloration; H.L. and A.V.D. provided theoretical analysis of mechanical properties and performed computer simulations; M.R., M.S., C.C., and D.A.I. conducted x-ray studies and data analysis; S.M. oversaw AFM studies; S.S.S. and A.V.D. were primary writers of the manuscript; and S.S.S. was the principal investigator. All authors discussed the results and provided feedback on the manuscript. Competing interests: M.V.-V. and S.S.S. are inventors on patent application US 62/585,124, submitted by the University of North Carolina at Chapel Hill, which covers synthesis of plastomers. Data and materials availability: All data needed to evaluate the conclusions in the paper are present in the paper and/or the supplementary materials. Data in the supplementary materials are presented in the form of tables and graphs. These include data on chemical composition and corresponding mechanical properties for each specific material studied in the paper.

\section{SUPPLEMENTARY MATERIALS}

www.sciencemag.org/content/359/6383/1509/suppl/DC1 Materials and Methods

Figs. S1 to S24

Tables S1 to S7

References (31-54)

Movies S1 and S2

18 November 2017; accepted 30 January 2018 10.1126/science.aar5308 


\section{Science}

\section{Chameleon-like elastomers with molecularly encoded strain-adaptive stiffening and coloration}

Mohammad Vatankhah-Varnosfaderani, Andrew N. Keith, Yidan Cong, Heyi Liang, Martin Rosenthal, Michael Sztucki, Charles Clair, Sergei Magonov, Dimitri A. Ivanov, Andrey V. Dobrynin and Sergei S. Sheiko

Science 359 (6383), 1509-1513.

DOI: $10.1126 /$ science.aar5308

\section{Active camouflage from a polymer}

Human skin is soft and compliant, but it can quickly become stiff when deformed to prevent injury. Chameleon skin can change color when the animal goes from a relaxed to an excited state. Although these properties can be captured individually in synthetic materials, the combination of different dynamic responses can be hard to control. Vatankhah-Varnosfaderani et al. created triblock copolymers of the ABA variety, where the A blocks have a linear structure and the B blocks are like bottlebrushes. When strained, these polymers stiffened like human skin and changed color, thus giving the materials a range of adaptive properties. Science, this issue p. 1509

ARTICLE TOOLS

SUPPLEMENTARY MATERIALS

RELATED CONTENT

REFERENCES

PERMISSIONS http://science.sciencemag.org/content/359/6383/1509

http://science.sciencemag.org/content/suppl/2018/03/28/359.6383.1509.DC1

http://robotics.sciencemag.org/content/robotics/3/18/eaat7175.full

This article cites 52 articles, 3 of which you can access for free http://science.sciencemag.org/content/359/6383/1509\#BIBL

http://www.sciencemag.org/help/reprints-and-permissions

Use of this article is subject to the Terms of Service

Science (print ISSN 0036-8075; online ISSN 1095-9203) is published by the American Association for the Advancement of Science, 1200 New York Avenue NW, Washington, DC 20005. The title Science is a registered trademark of AAAS.

Copyright (C) 2018 The Authors, some rights reserved; exclusive licensee American Association for the Advancement of Science. No claim to original U.S. Government Works 